\title{
The 'PAMELA anomaly' indicates a nearby cosmic ray accelerator
}

\author{
P. MERTSCH* and S. SARKAR \\ Rudolf Peierls Centre for Theoretical Physics, University of Oxford, \\ Oxford OX1 3NP, UK \\ *E-mail: p.mertsch1@physics.ox.ac.uk
}

\begin{abstract}
We discuss the recently observed 'excesses' in cosmic ray electron and positron fluxes which have been widely interpreted as signals of dark matter. By considering the production and acceleration of secondary electrons and positrons in nearby supernova remnants, we predict an additional, harder component that becomes dominant at high energies. The unknown spatial distribution of the supernova remnants introduces a stochastic uncertainty which we estimate analytically. Fitting the prediction for different source distributions to the total electron + positron flux measured by Fermi-LAT fixes all free parameters and allows us to 'postdict' the rise in the positron fraction seen by PAMELA. A similar rise in the $\mathrm{B} / \mathrm{C}$ ratio is predicted at high energies.
\end{abstract}

Keywords: dark matter indirect detection, galactic cosmic rays

\section{Introduction}

The recent findings of a rise in the positron fraction by PAMELA ${ }^{1}$ and an excess in the total $e^{+}+e^{-}$flux by Fermi-LAT ${ }^{2}$ have generated a lot of interest because these might be signals of dark matter annihilation or decay. However the expected signals are much smaller than those observed and models which attempt to circumvent this by invoking exotic new physics are increasingly constrained by $\gamma-$ ray and radio observations. It is thus important to investigate more prosaic astrophysical explanations, e.g. nearby pulsars $^{3,4}$ or supernova remnants (SNRs). In this context we wish to revisit the predictions of the standard SNR origin model of galactic cosmic rays (GCRs) wherein only primary electrons and nuclei are accelerated in SNRs by diffusive shock acceleration (DSA). ${ }^{5}$

We consider the recent proposal ${ }^{6}$ that some of the secondary $e^{+}$made in the SNRs are also accelerated by DSA. By accounting for the discrete distribution of the SNRs, we are then able to match the predicted fluxes to 
the measured $e^{+}+e^{-}$flux. Having thus fixed all the free parameters of our model we can make an independent 'postdiction' for the positron fraction which agrees remarkably well with the PAMELA data. We also predict a similar rise for the $\mathrm{B} / \mathrm{C}$ ratio as a definitive test of our model.

\section{The discreteness of sources}

Although SNRs can grow to become quite large (over $\sim 100 \mathrm{pc}$ in diameter) towards the end of their lifetime, they are still much smaller than the Kpc scales relevant for GCR propagation. Furthermore, the bulk of the particles accelerated by a SNR will be released towards the end of the Sedov-Taylor phase when they can no longer be confined by magnetic turbulence. We are therefore not dealing with a continuous distribution of sources within the galactic disk but with a large number of discrete sources, both in space and time. However, as we do not know the exact distribution, this discreteness introduces an uncertainty in the predicted total $e^{+}+e^{-}$flux. However, a generalised central limit theorem and the analytical form of the Green's function for the diffusion-energy loss problem can be used to infer the statistical properties of the flux, i.e. its average and quantiles. Our results are in accordance with earlier studies using the Monte Carlo approach. ${ }^{7-9}$

The flux from a source $i$ that injected $e^{-}$or $e^{+}$a time $t_{i}$ ago at a distance $L_{i}$ from the observer is given by the Green's function ${ }^{10} G_{\text {disk }}\left(E, L_{i}, t_{i}\right)$ of the diffusion equation: $J_{i}(E)=c /(4 \pi) G_{\text {disk }}\left(E, L_{i}, t_{i}\right)$. The flux $J(E)$ of $N$ identical sources at distances $\left\{L_{i}\right\}$ and times $\left\{t_{i}\right\}$ is just the sum of the individual fluxes,

$$
J=\sum_{i=1}^{N} J_{i}(E)=\frac{c}{4 \pi} \sum_{i=1}^{N} G_{\mathrm{disk}}\left(E, L_{i}, t_{i}\right) .
$$

As a function of the random variables $L$ and $t$, the Green's function $Z \equiv G_{\text {disk }}(E, L, t)$ is itself a random variable with probability density $f_{Z}$, expectation value $\mu_{Z}$ and standard deviation $\sigma_{Z}$. If the central limit theorem were applicable, the fluxes $J$ for different realisations of the same source density at a fixed energy $E$ would follow a normal distribution with mean $\mu_{J}=c /(4 \pi) N \mu_{Z}$ and standard deviation $\sigma_{J}=c /(4 \pi) \sqrt{N} \sigma_{Z}$.

In the case under consideration, $L$ and $t$ are assumed to be independent random variables with probability densities $f_{L}$ and $f_{t}$, respectively. We choose the sources to be distributed homogeneously in a ring with inner and outer radius $r_{1}$ and $r_{2}$, so $f_{L}=2 L /\left(r_{2}^{2}-r_{1}^{2}\right)$ for $r_{1} \leq r<r_{2}$ and 0 otherwise. For simplicity we also assume the source rate to be constant in time, i.e. $f_{t}=1 / t_{\max }$ for $0 \leq t \leq t_{\max }$ and 0 otherwise, up to a maximum time set by 
the minimum energy $E_{\min }$ under consideration: $t_{\max }=\left(b_{0} E_{\min }\right)^{-1}$. Here, $b_{0}$ is the normalisation of the energy loss rate of the $e^{-} / e^{+}: b(E)=b_{0} E^{2}$.

For $E>10 \mathrm{GeV}$ and $r_{1} \rightarrow 0$ (the distance to the nearest source is not limited physically) we find the expectation value:

$$
\mu_{J}=\frac{c}{4 \pi} \frac{1}{\sqrt{4 D_{0} b_{0}(1-\delta)}} \frac{N}{\pi r_{2}^{2}} \frac{Q_{0}}{t_{\max }} E^{-\gamma-1+(1-\delta) / 2} \frac{\Gamma\left(\frac{\gamma-1}{1-\delta}\right)}{\Gamma\left(\frac{2 \gamma-\delta-1}{2(1-\delta)}\right)} .
$$

where the interstellar diffusion coefficient $D_{0}=10^{28} \mathrm{~cm}^{2} \mathrm{~s}^{-1}$ at $E=1 \mathrm{GeV}$, and $\delta \simeq 0.6$ is its spectral index $\left(D \propto E^{\delta}\right)$. We set $b_{0}=10^{-16} \mathrm{GeV}^{-1} \mathrm{~s}^{-1}$ and $r_{2}=15 \mathrm{kpc}$ as is standard. The total number $N$ of sources that are needed to reproduce the (observed) number $\mathcal{N} \simeq 300$ of SNRs active in the Galaxy at any given time depends on the average lifetime of a SNR, $\tau_{\mathrm{SNR}}$, which is suggested to be $\sim 10^{4} \mathrm{yr},{ }^{11}$ hence $N=\mathcal{N} t_{\max } / \tau_{\mathrm{SNR}}=10^{6}$. With $Q_{0}=8.4 \times 10^{49} \mathrm{GeV}^{-1}$, the expectation value,

$$
E^{3} \mu_{J} \simeq 150 \mathrm{GeV}^{-1} \mathrm{~cm}^{-2} \mathrm{~s}^{-1} \mathrm{sr}^{-1},
$$

closely matches the featureless $E^{-3}$ spectrum measured by Fermi-LAT. ${ }^{2}$

Whereas the expectation value $\mu_{J}$ is well-defined, the variance $\sigma_{J}^{2}$ diverges because $f_{Z}(z)$ has a long power law tail. Nevertheless, instead of the central limit theorem, one can apply a generalised version ${ }^{12}$ which allows determination of the distribution of $J$ from the asymptotic behaviour of $f_{Z}(z)$ for $z \rightarrow \infty$. The distribution of $J$ is a so-called stable distribution ${ }^{13}$ - an asymmetric generalisation of a Gaussian which also exhibits a power law behaviour for large values of $J$. The uncertainty interval around the expectation value $\mu_{J}=c /(4 \pi) N \mu_{Z}$ can then be defined by quantiles of the stable distribution. The energy dependence of the quantiles turns out to be always harder than that of the expectation value, so the fluctuations are growing with energy. Figure 1 shows the uncertainty bands around the expectation value for the flux $\mu_{J}$, together with the expected fluxes from 50 random realisations of the above source distribution.

To calculate the fluxes of $e^{-}$and $e^{+}$at Earth, we perform a Monte Carlo calculation by considering a large number of realisations of randomly distributed sources according to a probability density function which reflects our present knowledge concerning the distribution of SNRs in the Galaxy. We emphasise that the better the expected flux of $e^{-}$and $e^{+}$from such a realisation of the source density matches the measured fluxes, the closer is the underlying distribution of sources likely to be to the actual one. We do not consider any variations between the SNRs but assume a prototypical 


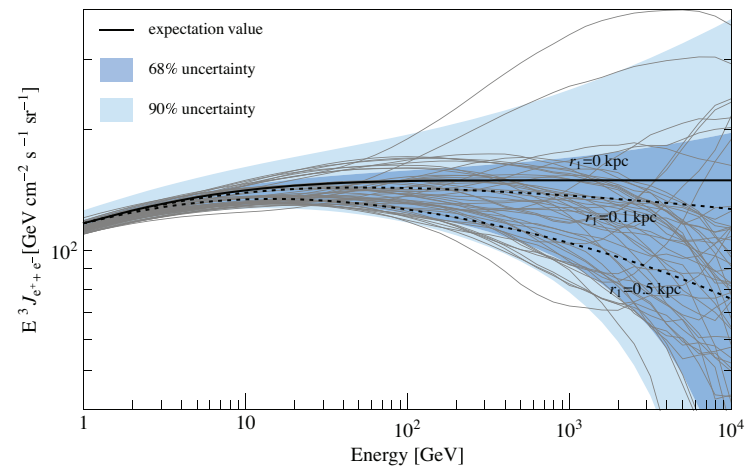

Fig. 1. Fluxes of high energy $e^{-}$from ensembles of sources uniformly distributed in a disk around the observer. The solid line denotes the expectation value for the sum of fluxes from $N$ discrete, transient sources while the dashed lines show the expectation values if the sources are limited to a ring with inner radius $r_{1}$ (normalised to the expectation value for $r_{1}=0$ at $1 \mathrm{GeV}$ ). The coloured bands quantify the fluctuations and contain, respectively, $68 \%$ and $90 \%$ of the calculated fluxes. The fluxes from 50 random realisations of an ensemble of $N$ individual sources are shown by the thin grey lines.

set of source parameters which we determine from a compilation of $\gamma$-ray SNRs, see Sec. 3. Of course all SNRs are not the same, however variations of the source parameters would only introduce additional fluctuations in the fluxes without altering their average.

For a more realistic distribution of source distances, we model the SNR density by a logarithmic spiral tracing the gas density (see Fig. 2) and also include the known radial distribution of SNRs in the Galaxy from radio surveys. We transform to a coordinate system centred on the Sun and integrate over azimuth such that our calculated probability density (Fig. 3) encodes the average surface density of SNRs at a particular distance.

\section{Primary electrons}

The injection of primary $e^{-}$by SNRs is parametrised as:

$$
R_{e^{-}}=R_{e^{-}}^{0}\left(\frac{E}{\mathrm{GeV}}\right)^{-\gamma} \mathrm{e}^{-E / E_{\text {cut }}} .
$$

The spectral index $\gamma$ and the cut-off energy $E_{\text {cut }}$ can be obtained from the spectral indices of SNRs in $\gamma$-rays as measured by Imaging Air Cerenkov Telescopes (IACTs), like HESS, MAGIC and VERITAS. (We assume the same spectral index for the hadronic and the electronic components, as predicted for DSA.) We have compiled ${ }^{14}$ a list of all SNRs detected by IACTs 


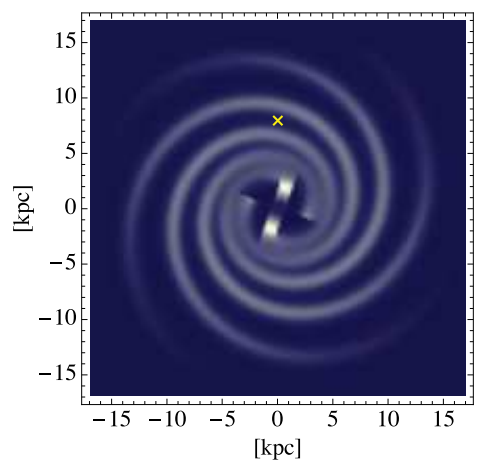

Fig. 2. The adopted distribution of SNRs in the Galaxy - the cross denotes the position of the Sun.

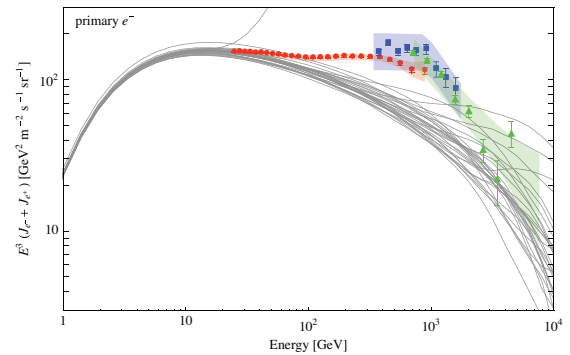

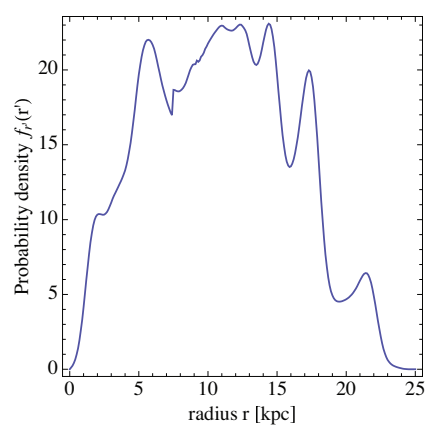

Fig. 3. The probability density for the distance of a SNR from the Sun.

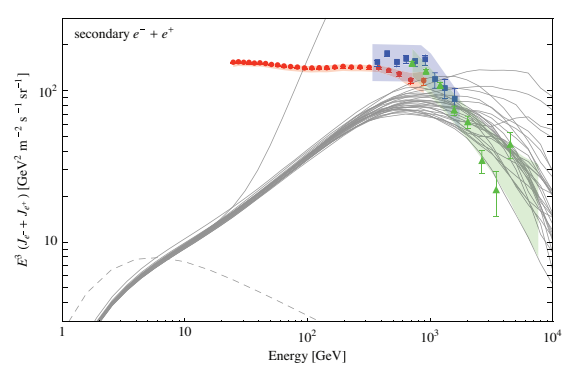

Fig. 4. Predicted spectra of $e^{-}$and $e^{+}$with data from Fermi-LAT ${ }^{2}$ (red circles) and HESS $^{17,18}$ (blue squares \& green triangles). Left: Primary $e^{-}$after propagation to Earth. Right: Secondary $e^{-}$and $e^{+}$from cosmic ray interactions, created during propagation (dashed line) and created during acceleration in SNRs (full lines).

and find the typical values to be $\gamma=2.4$ and $E_{\text {cut }}=20 \mathrm{TeV}$. The normalisation $R_{e^{-}}^{0}$ is determined by fitting the electron flux at Earth from our Monte Carlo computation to the preliminary measurement by PAMELA at $10 \mathrm{GeV} ;{ }^{15}$ the secondary fluxes can be neglected for this purpose. We find $R_{e^{-}}^{0}=1.8 \times 10^{50} \mathrm{GeV}^{-1}$ for $\gamma=2.4$ which corresponds to a total injection energy of $\int \mathrm{d} E E R_{e^{-}}(E) \simeq 7 \times 10^{47} \mathrm{erg}$. Solar modulation is treated in the spherical force-field approximation ${ }^{16}$ with a potential of $\phi=600 \mathrm{MV}$.

The primary electron fluxes on Earth from a large number of realisations of the source distribution are shown in the left panel of Fig. 4 and clearly exhibit a deficit with respect to the measurements by Fermi-LAT and HESS. 


\section{Additional positrons from the acceleration of secondaries}

It is usually assumed that secondary $e^{-}$and $e^{+}$are produced only through spallation (mainly on interstellar hydrogen and helium) during the galactic propagation of hadronic cosmic rays. At energies above $10 \mathrm{GeV}$, the spectrum of such secondaries after propagation should be softer than that of the primaries (by the power $\delta$, the spectral index of the diffusion coefficient), and the positron fraction should therefore be falling. We calculate secondary $e^{+}$from propagation of protons and nuclei following Ref. 19, using however the propagation parameters specified above.

It was recently suggested ${ }^{6}$ that the acceleration of secondary $e^{-}$and $e^{+}$in the cosmic ray sources, i.e. SNRs, could lead to a harder source spectrum of $e^{+}$that can explain the observed rise in the positron fraction. As the volume in which particles that participate in DSA grows with the diffusion coefficient $D_{\mathrm{Bohm}}(p) \propto p$, the fraction of the secondaries which can participate in DSA also increases linearly with momentum. The resulting source spectrum $R_{ \pm}$of secondary $e^{-}$and $e^{+}$is then a sum of two power laws, corresponding to the unaccelerated and the accelerated secondaries:

$$
R_{ \pm} \simeq R_{ \pm}^{0} p^{-\gamma}\left[1+\left(\frac{p}{p_{\text {cross }}}\right)\right]
$$

where the "cross-over" momentum, $p_{\text {cross }}$, satisfies

$$
D\left(p_{\text {cross }}\right)=\frac{3}{4} \frac{r u_{1}^{2} \tau_{\mathrm{SNR}}}{(\gamma+2)\left(1 / \xi+r^{2}\right)} .
$$

As has been noted, ${ }^{6}$ this mechanism is most efficient for old SNRs where field amplification by the shock wave is not very effective anymore. We therfore introduce a fudge factor $K_{\mathrm{B}}$ that parameterises the effect of the smaller field amplification on the otherwise Bohm-like diffusion coefficient in the SNR,

$$
D(E)=3.3 \times 10^{22} K_{\mathrm{B}}\left(\frac{B}{\mu \mathrm{G}}\right)^{-1}\left(\frac{E}{\mathrm{GeV}}\right) \mathrm{cm}^{2} \mathrm{~s}^{-1} .
$$

A break in the source spectrum occurs at $p_{\text {break }}$ because the growth of the acceleration zone is bounded by the physics size of the SNR. The source spectrum $R_{ \pm}$thus returns to a $p^{-\gamma}$ dependence around $p=p_{\text {break }}$. At even higher energies the secondary spectrum cuts off at the same $E_{\text {cut }}$ as the primary $e^{-}$(see Sec. 3).

Following Refs. 6,20 , the parameters are chosen to be: $u_{1}=0.5 \times$

$10^{8} \mathrm{~cm} \mathrm{~s}^{-1}, n_{\text {gas }, 1}=2 \mathrm{~cm}^{-3}, B=1 \mu \mathrm{G}$. Instead of fixing the normalisation of the injection spectrum ad hoc, we determine it from $\gamma$-ray observations 

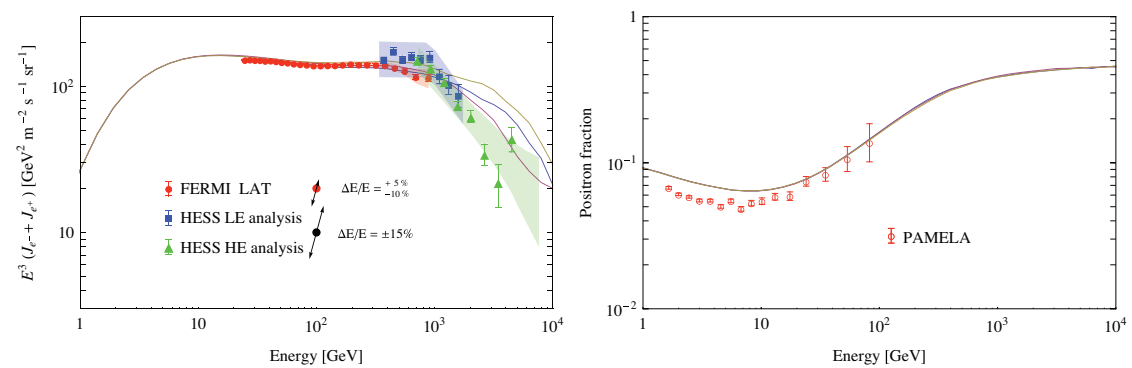

Fig. 5. Left: Predicted spectra of the total flux of primary and secondary $e^{-}$and $e^{+}$. Right: The predicted positron fraction after fixing all free parameters using the total $\left(e^{+}+e^{-}\right)$flux, compared with data from PAMELA. ${ }^{1}$

of SNRs. Knowing the cross-sections for the production of $\gamma$-rays (from the decay of neutral pions) and the production of secondary $e^{-}$and $e^{+}$(from the decay of charged pions), we determine the normalisation

$$
R_{+}^{0}=7.4 \times 10^{48}\left(\frac{\tau_{\mathrm{SNR}}}{10^{4} \mathrm{yr}}\right)\left(\frac{Q_{\gamma}^{0}}{5.7 \times 10^{33} \mathrm{~s}^{-1} \mathrm{TeV}^{-1}}\right) \mathrm{GeV}^{-1} .
$$

where $Q_{\gamma}^{0}$ is the observed typical luminosity of SNR in $\gamma$-rays as determined from the same compilation of data as above.

The right panel of Fig. 4 shows the expected flux of secondary $e^{-}$and $e^{+}$for 30 realizations of the possible distribution of SNRs in our Galaxy. Clearly this component can potentially match the high energy Fermi-LAT and HESS data. We also show the secondary $e^{-}$and $e^{+}$from the propagation of protons and nuclei, which is subdominant.

\section{Total electron-positron flux and positron fraction}

Figure 5 shows the total $\left(e^{+}+e^{-}\right)$flux obtained by adding the primary $e^{-}$and the secondary $e^{-}$and $e^{+}$(see. Fig. 4). As explained above, we selected just those (3) realisations of the source distribution which give the best fit to the measurements by Fermi-LAT and HESS. By fitting to the $\left(e^{+}+e^{-}\right)$flux we have also fixed the value for the diffusion coefficient near the SNR shock wave (which determines the ratio of the accelerated to the unaccelerated secondaries) to about 15 times the Bohm value i.e. $K_{B} \simeq 15$.

Having thus fixed all free parameters we can make an independent prediction for the positron fraction which is shown in the right panel of Fig. 5. It is in very good agreement with the PAMELA measurements above $\gtrsim 10 \mathrm{GeV}$; we do not expect to match the data below $10 \mathrm{GeV}$ be- 


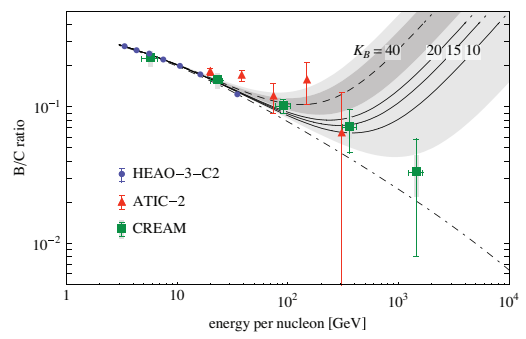

Fig. 6. The predicted B/C ratio in cosmic rays (from Ref. 22) for the 'leaky box model' with production of secondaries during propagation only (dot-dashed line), and including production and acceleration of secondaries in nearby SNRs (solid lines) for values of the diffusion coefficient near the shock wave which best fit the $e^{ \pm}$spectrum (see Sec. 5). The dashed line corresponds to the value of the diffusion co-efficient required to fit ATIC-2 data on $\mathrm{Ti} / \mathrm{Fe}$ (from Ref. 22), along with its $1 \sigma$ and $2 \sigma$ error bands. The data points are from HEAO-3-C2 (circles), ${ }^{24}$ ATIC-2 (triangles) ${ }^{25}$ and CREAM (squares). ${ }^{26}$

cause our analytic approach neglects complications such as convection and reacceleration in the ISM which can be important at these energies.

\section{Nuclear secondary-to-primary ratios}

The acceleration of secondaries described above modifes not only the spectra of secondary $e^{-} \& e^{+}$but also of other charged secondaries. This provides a direct test of this explanation for the rise in the positron fraction, e.g. a rise in the antiproton-to-proton ratio is also predicted ${ }^{20}$ (consistent with the measurements by PAMELA ${ }^{21}$ so far). We have argued ${ }^{22}$ that nuclear secondary-to-primary ratios can be used to not only test this model but also to discriminate against alternative explanations such as dark matter annihilation/decay or nearby pulsars which cannot affect nuclear abundances.

We have calculated the rise in nuclear secondary-to-primary ratios expected from the acceleration of secondaries, in particular, we have used recent ATIC-2 data on the titanium-to-iron ratio which exhibit a rise around $100 \mathrm{GeV} / \mathrm{n}$ to fix the diffusion coefficient. We find a 'fudge factor' of $K_{B} \simeq 40$ is necessary, albeit with large error bars due to the limited statistics of the ATIC-2 data. Again, we can make an independent prediction for a different secondary-to-primary ratio, namely boron-to-carbon (B/C). Our prediction, together with the canonical expectation for a purely secondary origin through spallation in the ISM, are shown in Fig. 6 with a selection of data. Currently, B/C is being measured by PAMELA, and it is also a prime goal of the upcoming AMS-02 mission. ${ }^{23}$ 


\section{Conclusion}

We have discussed the recently measured excesses in the $e^{+}$fraction and in the total $e^{+}+e^{-}$flux in galactic cosmic rays. The fluctuations induced by the discreteness of the cosmic ray sources has been estimated assuming these to be SNRs. However when the source spectral index is estimated from $\gamma$-ray observations of SNRs, the expected total $e^{+}+e^{-}$flux at Earth is deficient. Adding the flux of secondary $e^{-}$and $e^{+}$produced and accelerated in nearby SNRs brings the model prediction back in agreement with the measurements and naturally implies a rise in the $e^{+}$fraction. A crucial test of this idea is to determine if nuclear secondary-to-primary ratios, e.g. B/C, also increase with energy. Such nearby SNRs ought also to be detected by the IceCube neutrino observatory in a few years. ${ }^{22}$ This would be the first direct astronomical identification of the sources of galactic cosmic rays.

\section{References}

1. O. Adriani et al., Nature 458, 607 (2009).

2. A. A. Abdo et al., Phys. Rev. Lett. 102, p. 181101 (2009).

3. F. Aharonian, A. Atoyan and H. Volk, Astron. Astrophys. 294, L41 (1995).

4. D. Hooper, P. Blasi and P. D. Serpico, JCAP 0901, p. 025 (2009).

5. M. A. Malkov and L. O'C Drury, Rept. Prog. Phys. 64, 429 (2001).

6. P. Blasi, Phys. Rev. Lett. 103, p. 051104 (2009).

7. M. Pohl and J. A. Esposito, Astrophys. J. 507, p. 327 (1998).

8. A. W. Strong and I. V. Moskalenko (2001).

9. S. P. Swordy, in Proceedings of the 28th ICRC, Tsukuba, Japan, 2003.

10. V. L. Ginzburg et al. Amsterdam, Netherlands: North-Holland (1990) 534 p.

11. S. P. Reynolds, Annu. Rev. Astron. Astrophys. 46, p. 89 (2008).

12. B. V. Gendenko and A. N. Kolmogorov, Limit Distributions for Sums of Independent Random Variables (Addison-Wesley, Cambridge, MA, USA, 1954).

13. J. P. Nolan, Stable Distributions - Models for Heavy Tailed Data (Birkhäuser, Boston, 2010).

14. M. Ahlers, P. Mertsch and S. Sarkar, Phys. Rev. D80, p. 123017 (2009).

15. E. Mocchiutti, (2009), Talk at 2nd RICAP, Rome, 13-15 May 2009.

16. L. J. Gleeson and W. I. Axford, Astrophys. J. 154, p. 1011 (1968).

17. F. Aharonian et al., Phys. Rev. Lett. 101, p. 261104 (2008).

18. F. Aharonian et al., Astron. Astrophys. 508, p. 561 (2009).

19. T. Delahaye et al., Astron. Astrophys. 501, 821 (2009).

20. P. Blasi and P. D. Serpico, Phys. Rev. Lett. 103, p. 081103 (2009).

21. O. Adriani et al., Phys. Rev. Lett. 102, p. 051101 (2009).

22. P. Mertsch and S. Sarkar, Phys. Rev. Lett. 103, p. 081104 (2009).

23. AMS Webpage, http://ams.cern.ch/.

24. J. J. Engelmann et al., Astron. Astrophys. 233, 96(July 1990).

25. A. D. Panov et al. (2007), Proc. 30th ICRC, Merida.

26. H. S. Ahn et al., Astropart. Phys. 30, 133 (2008). 